\title{
Analyzing the Characteristics of Simulated E-field Environment of Reverberation Chamber
}

\author{
Kaifu Ji a, ${ }^{*}$, Guanghui Wei, Xiaodong Pan and Haojiang Wan \\ Research Institute of Static Electricity \& Electromagnetic Protection, Ordnance Engineering \\ College, Shijiazhuang 050003, China \\ ajikaifu@sohu.com
}

Keywords: Reverberation Chamber; Degree of Fitting; KS Test.

\begin{abstract}
This paper analyzes the statistical characteristics of the ideal reverberation chamber environment, and evaluate the simulated reverberation room environment at different frequencies based on the KS test, to test the degree of fitting between the simulated E-field environment and theoretical reverberation chamber environment. The results show that the probability of accepting the original hypothesis in the simulation is high, the model can be used in the simulation analysis of the reverberation chamber.
\end{abstract}

\section{Introduction}

Reverberation chamber is a new radiation sensitivity test site with unique design principle. It can form statistically uniform, random polarization, isotropic electromagnetic environment, and have the features of simple test process, low power to produce high field strength, construction low cost and so on[1-3]. Compared with the traditional test sites, reverberation room has become the study concern of the majority of scholars in recent years for its significant advantages. With the continuous development of numerical calculation technology and the rapid increase of computer running speed, the simulation calculation of reverberation chamber has been widely applied in the cavity electromagnetic environment analysis and practical engineering construction.

The established simulation models of the reverberation chamber both at home and abroad mainly focus on the three-dimensional simulation model with the same size as the actual reverberation chamber, which is convenient to instruct the construction, optimization and parameter evaluation of the actual reverberation chamber[4-5]. Due to the statistical uniformity of the E-field distribution in the reverberation chamber, the statistical E-field distribution of the total positions of the stirer is focused on the radiation sensitivity test. The specific E-field distribution corresponding to single position of stirer is insignificant. Reverberation indoor radiation sensitivity test for the purpose of establishing a simulation model, just to build the actual reverberation chamber model consistent with the E-field environment. Therefore we just need to construct the simulated E-field environment which is the same as the E-field environment in actual reverberation chamber. In order to demonstrate the feasibility of simulating the reverberation room environment, the plane wave superposition theory is applied to the reverberation chamber, and the suitable simulation calculation method and simulation software are used to construct the reverberation chamber E-field model with finite random plane wave superimposed simulation. And we use KS test to analyse the statistical distribution of the the model and the actual reverberation chamber environment, to measure the degree of consistency between the simulated reverberation chamber E-field environment and the ideal reverberation chamber E-field environment.

\section{Simulation}

Franco Moglie and Anna Pia use FDTD method to construct a simulated reverberation chamber E-field environment based on the theory of D.A.Hill, and vertify the correctness of the model by comparing the experimental results in the simulated environment with the test results in actual reverberation chamber E-field environment[6-7]. In this paper, we use plane wave superposition 
principle to construct simulated reverberation chamber electric E-field environment. And the simulation software FEKO is used to set the parameters of the plane wave, which can ensure the accuracy of the premise, and significantly improve the efficiency of simulation calculations.

There are six parameters for each incident plane plane, which are the angle of pitch $\theta$, the direction angle $\varphi$, the polarization direction angle $\alpha$, the phase angle $\beta$, the E-field strength $E$, the frequency $f$. In order to obtain a uniform E-field environment, the E-field strength and frequency of each plane wave are consistent, and the polarization angle and phase angle are evenly distributed in $[0,2 \pi]$. In the spherical coordinate system, the differential expression of the solid angle is:

$$
d \Omega=\sin \theta d \theta d \varphi=d(\cos \theta) d \varphi
$$

To ensure that the simulated E-field environment is statistical uniform E-field,the parameters of the plane wave are set as Table 1:

Tab. 1 parameters setting distribution of random plane waves

\begin{tabular}{|c|c|}
\hline parameter & distribution \\
\hline$\theta$ & $\arccos u$ \\
\hline$\varphi$ & $U(0,2 \pi)$ \\
\hline$\alpha$ & $U(0,2 \pi)$ \\
\hline$\beta$ & $U(0,2 \pi)$ \\
\hline
\end{tabular}

Constructing the simulated reverberation chamber E-field environment with $\mathrm{N}$ column plane waves superposition, The effect of a single superposition is equivalent to the E-field distribution at one stirrer position, The simulated $\mathrm{M}$ times can be equivalent to the E-field distribution under the $\mathrm{M}$ stirrer positions in the reverberation chamber.

\section{Theoretical Analysis}

In this section,we analyse the thearetical distribution of E-field in reverberation chamber. When the stirrer in the reverberation chamber is fully stirred for one cycle, and uniform E-field environment in the cavity is formed. The rectangular E-field component of any positon in the test area can be expressed by the following formula:

$$
\left|E_{j}\right|=\sqrt{E_{j r}^{2}+E_{j i}^{2}}
$$

$E_{j}$ represents either rectangular component of the three directions $E_{x}, E_{y}, E_{z}$, in the Cartesian coordinate system, $E_{j r}$ and $E_{j i}$ represent the real and imaginary parts of $E_{j}$. The total E-field strength module value at any position can be expressed as:

$$
|E|=\sqrt{E_{x}^{2}+E_{y}^{2}+E_{z}^{2}}=\sqrt{E_{x r}^{2}+E_{x i}^{2}+E_{y r}^{2}+E_{y i}^{2}+E_{z r}^{2}+E_{z i}^{2}}
$$

In ideal reverberation chamber E-field environment, $E_{j r}$ and $E_{j i}$ obey the normal distribution :

$$
\begin{gathered}
f\left(E_{j r}\right)=\frac{1}{\sqrt{2 \pi} \sigma} e^{-E_{j r}^{2} / 2 \sigma^{2}} \\
f\left(E_{j i}\right)=\frac{1}{\sqrt{2 \pi} \sigma} e^{-E_{j i}^{2} / 2 \sigma^{2}}
\end{gathered}
$$

(4)and (5) show the probability density function(PDF) of $E_{j r}$ and $E_{j i}$. Therefore, the PDF of any rectangular E-field component in the working area can be expressed as:

$$
f\left(E_{j}\right)=\frac{E_{j}}{\sigma^{2}} \cdot e^{-\frac{E^{2}}{2 \sigma^{2}}}, E_{j}>0
$$

It is obvious that any rectangular E-field component obeys the Rayleigh distribution. We can derive the cumulative distribution function(CDF) [8] of rectangular E-field component from quadraturing (6), as the (7) shows: 


$$
F\left(E_{j}\right)=\int_{-\infty}^{E_{j}} f(E) \mathrm{d} E=\int_{0}^{E_{j}} \frac{E}{\sigma^{2}} \cdot e^{-\frac{E^{2}}{2 \sigma^{2}}} \mathrm{~d} E=1-e^{-\frac{E_{j}}{2 \sigma^{2}}}
$$

\section{KS Test}

To examine the difference between simulation results and ideal cumulative distribution function of E-field strength, we need to select the appropriate hypothesis test method to quantitatively describe the degree of fitting. Commonly used methods include the Pearson $\chi^{2}$ criterion and KolmogorovSmirnov (KS) test. The former one is generally used in the test with big simple size, the KS test is available for test with small simple size [9]. As for the number of agitator stepping positions is relatively small, we select KS test to examine the fitting degree. The KS test is a nonparametric test based on CDF, this method regards the ture distribution function $F_{n}(x)$ as the empirical distribution function and regards $F_{n}^{*}(x)$ as theoretical distribution function, then calculate the maximum difference between the corresponding sample values and consider it as the test statistic to measure the degree of difference between the two distribution. The calculation formula is as followed:

$$
D_{n}=\sup _{-\infty<x<+\infty}\left|F_{n}^{*}(x)-F_{n}(x)\right|
$$

For any sample value, the smaller $D_{n}$ indicates the smaller difference betewwn empirical distribution function and the theoretical distribution function. Set the confidence level to $95 \%$, that is $\alpha=5 \%$, then look up table according to $\alpha$ and simple size to see the critical value $D_{a}$. If $D_{n} \geq D_{a}$, that means the original hypothesis is invalid, which is $F(x) \neq F_{0}(x)$, can be represented as $\mathrm{H}=1$; on the contrary, if $D_{n}<D_{a}$, that means the original hypothesis is valid, which is $F(x)=F_{0}(x)$, can be represented as $\mathrm{H}=0$. And parameter $\mathrm{P}$ is introduced in $\mathrm{KS}$ test to characterize the probability of occurrence of sample observations when the original hypothesis is accepted, the larger $\mathrm{P}$ value represent the greater the probability that the original hypothesis occurs.

The 108 rectangular E-field components of 36 sampling positions in the working area of the simulated reverberation chamber environment were tested and the statistical characteristics at 111 test frequencies range from $80 \mathrm{MHz}$ to $300 \mathrm{MHz}$ were investigated. The result is as Fig. 2 shows:

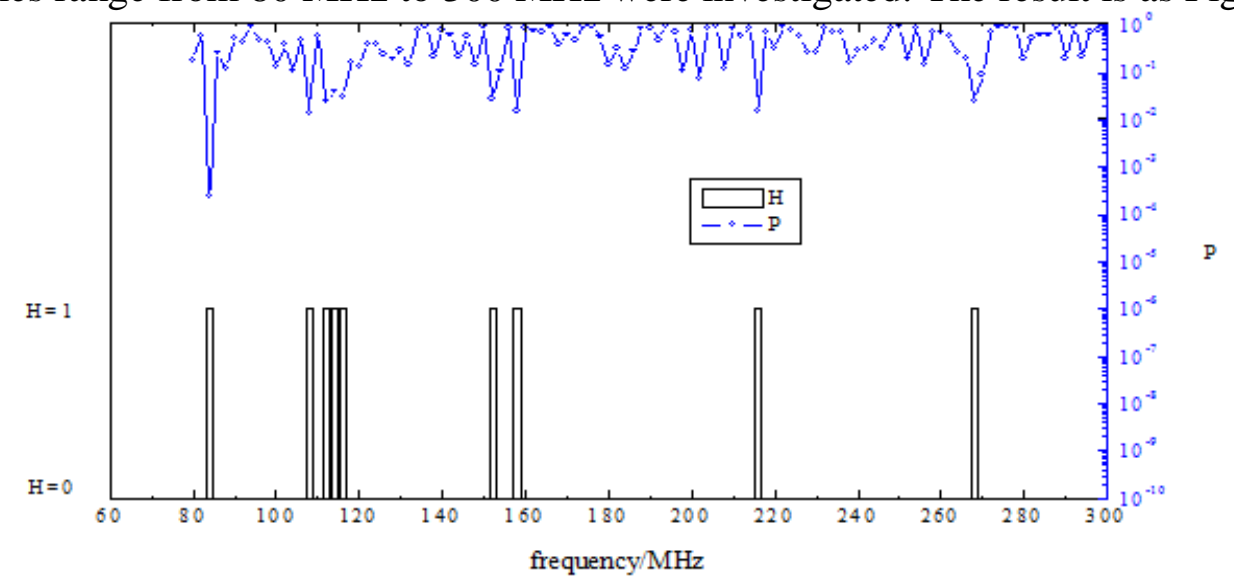

Fig. 2 KS test of statiscal distribution of simulated RC environment

In 9 test frequencies, the original hypothesis is rejected, accounting for $8.13 \%$ of the total number. And with the increase of frequency, the probability of rejecting original hypothesis is lower, and the mean value of $\mathrm{P}$ is greater, which means the distribution of rectangular E-field component of simulated reverberation chamber is closer to theoretical distribution.

It is concluded that the statistical characteristics of simulated E-field environment is great, the model can be used in the simulation analysis of the reverberation chamber. 


\section{References}

[1] Micheli D, Barazzetta M, Moglie F, et al. Power boosting and compensation during ota testing of a real $4 \mathrm{~g}$ lte base station in reverberation chamber[J]. IEEE Transactions on Electromagnetic Compatibility, 2015, 57(4): 623-634.

[2] Hussain A, Por Einarsson B, Kildal P S. Mimo ota testing of communication system using sdrs in reverberation chamber [measurements corner][J]. IEEE Antennas and Propagation Magazine, 2015, 57(2): 44-53.

[3] Sorrentino A, Gifuni A, Ferrara G, et al. Mode-stirred reverberating chamber autocorrelation function: Model, multifrequency measurements and applications[J]. Science, Measurement \& Technology, IET, 2015, 9(5): 547-554.

[4] Yang Z, Guanghui W, Yaozhong C, et al. Acceleration technique of modeling lossy reverberation chamber using fdtd method based on quality factor[J]. Antennas and Wireless Propagation Letters, IEEE, 2015(14):686-689.

[5] Yaozhong C, Guanghui W, Song W, et al. Fast analysis of reverberation chamber using fdtd method and matrix pencil method with new criterion for determining the number of exponentially damped sinusoids[J]. Electromagnetic Compatibility, IEEE Transactions on, 2014, 56(3): 510519.

[6] Hill D A. Plane Wave Integral Representation for Fields in Reverberation Chambers[J]. IEEE Transactions on Electromagnetic Compatibility, 1998, 40(3): 209-217.

[7] Franco Moglie, Anna Pia Pastore. FDTD analysis of plane wave superpositionto simulate susceptibility tests in reverberation chambers[J]. IEEE Transactions on Electromagnetic Compatibility, 2006, 48(1): 195-202.

[8] Yuhong Nie, Haigeng Chen. The calculation of cumulative distribution function of water vapor using modified bandwidth[J]. Journal of Thermal Science, 2002, 11(1):16-20.

[9] Wang F, Wang X. Fast and robust modulation classification via Kolmogorov-Smirnov test[J]. IEEE Transactions on Communications, 2010, 58(8):2324-2332. 